\title{
IRIS and SDO Observations of Solar Jetlets Resulting from Network-edge Flux Cancelation
}

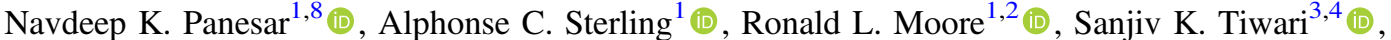 \\ Bart De Pontieu ${ }^{3,5,6}$ (iD), and Aimee A. Norton ${ }^{7}$ (D) \\ ${ }^{1}$ NASA Marshall Space Flight Center, Huntsville, AL 35812, USA; navdeep.k.panesar@nasa.gov, alphonse.sterling@nasa.gov \\ ${ }^{2}$ Center for Space Plasma and Aeronomic Research (CSPAR), UAH, Huntsville, AL 35805, USA \\ ${ }^{3}$ Lockheed Martin Solar and Astrophysics Laboratory, Org. A021S, Bldg. 252, 3251 Hanover St., Palo Alto, CA 94304, USA \\ ${ }^{4}$ Bay Area Environmental Research Institute, NASA Research Park, Moffett Field, CA 94035, USA \\ ${ }^{5}$ Rosseland Centre for Solar Physics, University of Oslo, P.O. Box 1029 Blindern, NO-0315 Oslo, Norway \\ ${ }^{6}$ Institute of Theoretical Astrophysics, University of Oslo, P.O. Box 1029 Blindern, NO-0315 Oslo, Norway \\ 7 W. W. Hansen Experimental Physics Laboratory, Stanford University, Stanford, CA 94305, USA \\ Received 2018 August 31; revised 2018 November 2; accepted 2018 November 7; published 2018 November 21
}

\begin{abstract}
Recent observations show that the buildup and triggering of minifilament eruptions that drive coronal jets result from magnetic flux cancelation at the neutral line between merging majority- and minority-polarity magnetic flux patches. We investigate the magnetic setting of 10 on-disk small-scale UV/EUV jets (jetlets, smaller than coronal X-ray jets but larger than chromospheric spicules) in a coronal hole by using IRIS UV images and SDO/AIA EUV images and lineof-sight magnetograms from $S D O / \mathrm{HMI}$. We observe recurring jetlets at the edges of magnetic network flux lanes in the coronal hole. From magnetograms coaligned with the IRIS and AIA images, we find, clearly visible in nine cases, that the jetlets stem from sites of flux cancelation proceeding at an average rate of $\sim 1.5 \times 10^{18} \mathrm{Mx} \mathrm{hr}^{-1}$, and show brightenings at their bases reminiscent of the base brightenings in larger-scale coronal jets. We find that jetlets happen at many locations along the edges of network lanes (not limited to the base of plumes) with average lifetimes of 3 minutes and speeds of $70 \mathrm{~km} \mathrm{~s}^{-1}$. The average jetlet-base width $(4000 \mathrm{~km})$ is three to four times smaller than for coronal jets $(\sim 18,000 \mathrm{~km})$. Based on these observations of 10 obvious jetlets, and our previous observations of larger-scale coronal jets in quiet regions and coronal holes, we infer that flux cancelation is an essential process in the buildup and triggering of jetlets. Our observations suggest that network jetlet eruptions might be small-scale analogs of both larger-scale coronal jets and the still-larger-scale eruptions producing CMEs.
\end{abstract}

Key words: magnetic fields - Sun: chromosphere - Sun: corona

Supporting material: animations

\section{Introduction}

Solar jets are short-lived, collimated, transient events frequently observed in the solar atmosphere (Shibata et al. 1992; Innes et al. 2011; Raouafi et al. 2016). Jets of all sizes, down to and including spicules (De Pontieu et al. 2007; Sterling \& Moore 2016), plausibly power the heating of the non-active-region global corona (Moore et al. 1999; De Pontieu et al. 2011). Coronal jets (CJs) occur in various solar environments (e.g., in active regions, quiet-Sun regions, and coronal holes), and launch plasma high into the corona (Wang et al. 1998; Moore et al. 2015; Panesar et al. 2016a).

Recent observations show that CJs are frequently driven by the eruption of a minifilament (Sterling et al. 2015; also see Hong et al. 2011; Shen et al. 2012; Adams et al. 2014) and a jet bright point (JBP) appears under the minifilament as it erupts to drive the jet. Minifilaments are seen to rise earlier $\left(<_{1 \mathrm{~min}}\right)$ than the manifestation of JBP (Moore et al. 2018). The JBP is a miniature version of the flare arcades that grow over polarity inversion lines (PILs) in the wake of larger-scale filament eruptions that drive coronal mass ejections (CMEs). Thus, CJs are analogous to typical larger-scale solar eruptions (Zirker 1989; Martin 1973).

Recently, we investigated the triggering mechanism of 10 quiet-region (Panesar et al. 2016b, 2017) and 13 coronal-hole (Panesar et al. 2018) CJs and found that CJs in these regions are

\footnotetext{
8 Also, Visiting Scholar at W. W. Hansen Experimental Physics Laboratory, Stanford University, Stanford, CA 94305, USA.
}

driven by the eruption of a minifilament, and that the minifilament magnetic field is built (minifilaments exist for periods ranging from $1.5 \mathrm{hr}$ to 2 days prior to their eruption) and triggered by magnetic flux cancelation at the PIL underneath the minifilament.

Using Solar Dynamic Observatory (SDO)/Atmospheric Imaging Assembly (AIA; Lemen et al. 2012) images, Raouafi \& Stenborg (2014) found jet-like features at flux cancelation sites at the bases of plumes. They named these features jetlets, because they are smaller than typical CJs. In the region we study here, we found jetlets at the base of plumes, but we also found identical-looking features outside of plumes too; we call all of these features jetlets. In this study we characterize jetlet properties, compare their properties to CJs, and try to determine whether they are small-scale counterparts to CJs.

We investigate in detail the magnetic setting of 10 on-disk solar UV/EUV network jetlets and examine their physical properties using Interface Region Imaging Spectrograph (IRIS; De Pontieu et al. 2014) and SDO data. We find that flux cancelation is the cause of most of our jetlets, and that they often occur at the edges of network lanes away from the bases of plumes. Raouafi \& Stenborg (2014) only reported jetlets that happened at the base of plumes; we find that jetlets are more wide spread in network regions, not limited to only the base of plumes.

\section{Instrumentation and Data}

IRIS provides simultaneous images (slit-jaw) and spectra of the solar atmosphere with high spatial resolution of 0 ". 16 pixel $^{-1}$ and 
cadence as high as $1.5 \mathrm{~s}$ in four different passbands (C II 1330, Si IV 1400, Mg II k 2796, and Mg II wing $2830 \AA$ A; De Pontieu et al. 2014). For our investigation, we used C II and Si IV slit-jaw images (SJIs) having a cadence of 2 minutes.

We also used $171 \AA$ extreme ultraviolet (EUV) images from $S D O /$ AIA to view the coronal-temperature plasma in the jetlets. To study the magnetic field evolution of the jetlet-base region, we employed line-of-sight magnetograms from the SDO/Helioseismic and Magnetic Imager (HMI; Schou et al. 2012).

For our analysis, we randomly selected an on-disk coronal hole network region covered by IRIS on 2016 March $19^{9}$ during 18:19-21:48UT. During the $3.5 \mathrm{hr}$ of IRIS coverage, we find five jetlets at five different locations/PILs. We intentionally avoided CJs for this study (e.g., Panesar et al. 2016b). AIA images show some plumes in the coronal hole. We find three jetlet locations/PILs at the base of plumes (Raouafi \& Stenborg 2014; Avallone et al. 2018) and two jetlet locations/PILs away from plumes (Figure 1). None of these jetlets were covered by the IRIS spectral slit.

Simultaneously, we study the same network region using AIA $171 \AA$ images for $24 \mathrm{hr}$ centered on the IRIS coverage period to see if there are more jetlets from the same PILs. We find five more jetlets within the IRIS field of view (FOV; Figure 1) but outside the IRIS observation time. All 10 jetlets and their measured parameters are listed in Table 1. Out of the 10 jetlets seen in AIA, only 5 of them (Table 1) were observed by IRIS.

\section{Results \\ 3.1. Overview}

Figure 1 shows the coronal-hole region observed by IRIS, $S D O$, and HMI. Jetlet locations are marked by red boxes in Figure 1(c). In this coronal hole, positive flux is in the majority, and 9 of the 10 jetlets originate from the PIL between a majority-polarity network flux lane and a merging minoritypolarity flux patch.

Jetlets from locations $\mathrm{A}, \mathrm{B}$, and $\mathrm{C}$ occur at the base of plumes, but the other two jetlet locations (D and E) are away from plumes. More generally, AIA $171 \AA$ images show that jetlets are very common in network regions. They occur at the edges of the magnetic network lanes, and are rather frequent; we find $\sim 40$ jetlets in $24 \mathrm{hr}$ within the AIA FOV of Figure 1(b). For the present study, we examine only those network-edge locations at which at least one jetlet was caught in IRIS images. Next, we present jetlets C3, D1 and E1 in detail.

\subsection{Jetlets from Region $C$}

Figures 2(a)-(c) and 3(a)-(c) show jetlet C3. Figures 4(a), (b) display the photospheric magnetic field of the jetlet-base region. The three jetlets $\mathrm{C} 1-\mathrm{C} 3$ were homologous, in that they originated from the same PIL and had similar structure (Dodson-Prince \& Bruzek 1977). During jetlet C3's onset, at 19:15 UT, we observe brightenings at its base (Figure 2(b)). Concurrently, brightenings also appear in AIA $171 \AA$ images (Figure 3(b), MOVIE2a). These base brightenings appear to

\footnotetext{
9 http://www.lmsal.com/hek/hcr?cmd=view-event\&event-id=ivo $\% 3 \mathrm{~A} \%$ 2F\%2Fsot.lmsal.com\%2FVOEvent\%23VOEvent_IRIS_20160319_181933_ 3601112078_2016-03-19T18\%3A19\%3A332016-03-19T18\%3A19\% 3A33.xml
}

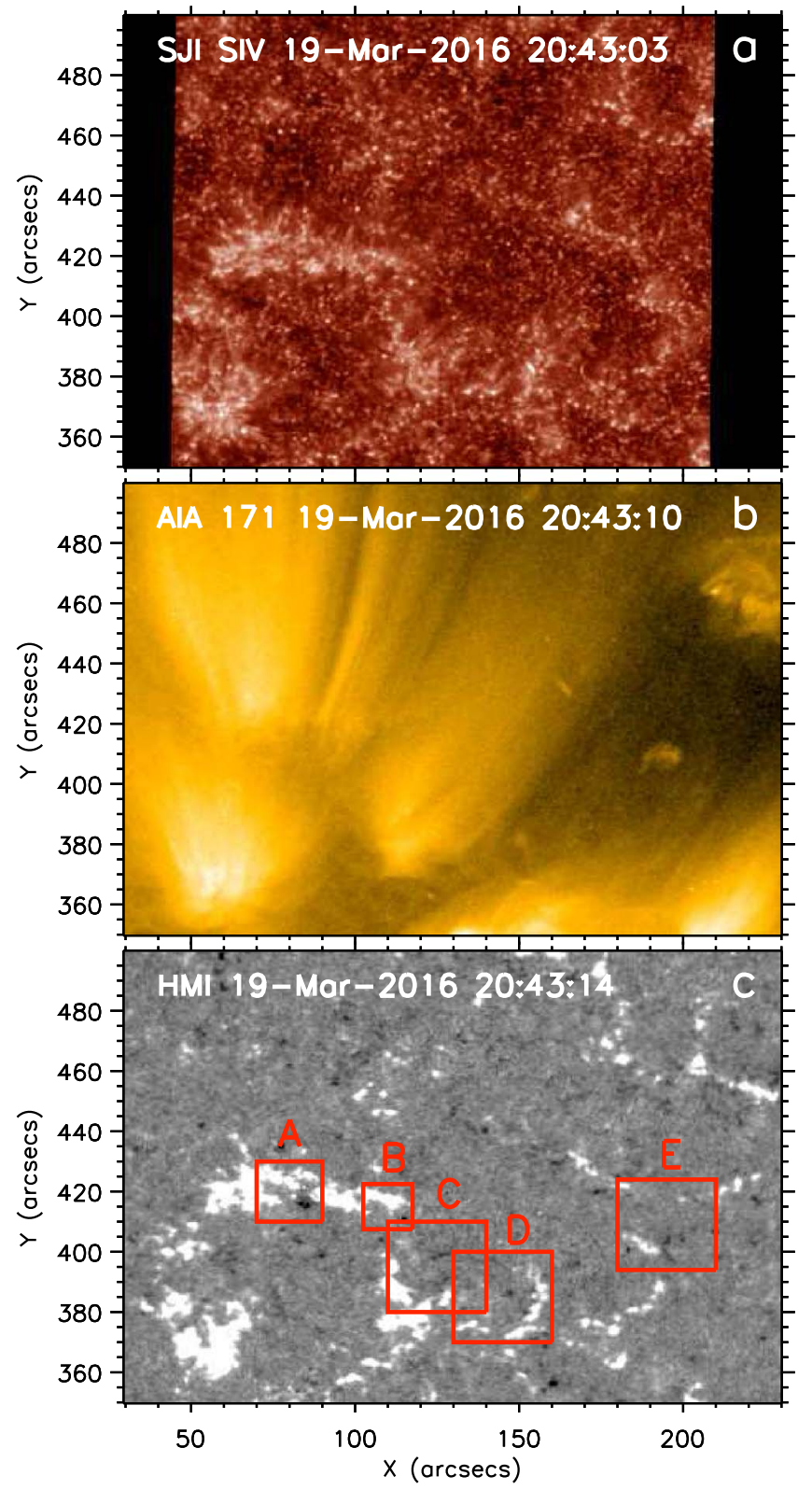

Figure 1. Locations of the 10 jetlets of Table 1: Panel (a) shows an IRIS Si IV SJI of the coronal-hole region. Panels (b) and (c) show an AIA $171 \AA$ image and an HMI magnetogram of the same region. The red boxes show the FOVs analyzed in detail and multiple jetlets appear within these FOVs.

include a miniature version of a JBP. After the start of base brightening, the spire extends outward with an average speed of $120 \pm 50 \mathrm{~km} \mathrm{~s}^{-1}$.

The IRIS and AIA animations show possible twisting motion in the jetlet spire over 19:17 UT-19:21 UT (MOVIE2a; Figures 2(b), (c) and 3(b), (c)). Such twisting motion perhaps resulted from external reconnection (Sterling et al. 2015) of a miniature erupting flux rope as inferred for larger CJs (Moore et al. 2015). The earlier two jetlets from this region do not show twisting motion.

HMI magnetograms (Figures 4(a), (b)) show that the jetlets originate from the PIL between a majority-polarity (positive) network flux lane and a merging minority-polarity (negative) flux patch (MOVIE3a). Figure 4(c) shows the negative-flux plot of the jetlet-base region over four hours. We only 
Table 1

Location and Measured Parameters of Observed Jetlets

\begin{tabular}{|c|c|c|c|c|c|c|c|c|c|c|c|c|}
\hline $\begin{array}{l}\text { Jetlet }^{\mathrm{a}} \\
\text { Location }\end{array}$ & $\begin{array}{l}\text { No. of }{ }^{b} \\
\text { Jetlets }\end{array}$ & $\begin{array}{l}\text { Time }^{\mathrm{c}} \\
\text { (UT) }\end{array}$ & $\begin{array}{c}I R I S \\
\text { Coverage }\end{array}$ & $\begin{array}{l}\text { Spire Length }^{\mathrm{d}} \\
\text { IRIS }(\mathrm{km})\end{array}$ & $\begin{array}{l}\text { Spire Length }^{\mathrm{d}} \\
\text { AIA }(\mathrm{km})\end{array}$ & $\begin{array}{l}\text { Spire Width }^{\mathrm{e}} \\
\text { IRIS }(\mathrm{km})\end{array}$ & $\begin{array}{l}\text { Spire Width }{ }^{\mathrm{e}} \\
\text { AIA }(\mathrm{km})\end{array}$ & $\begin{array}{c}\text { Jetlet } \\
\text { Speed }^{\mathrm{f}} \\
\left(\mathrm{km} \mathrm{s}^{-1}\right)\end{array}$ & $\begin{array}{c}\text { Jetlet } \\
\text { Dur. }^{\mathrm{g}} \\
\text { (minutes) }\end{array}$ & $\begin{array}{c}\text { Jetlet base }^{\mathrm{h}} \\
\text { AIA }(\mathrm{km})\end{array}$ & $\begin{array}{l}\text { Jetlet base }^{\mathrm{h}} \\
\text { IRIS }(\mathrm{km})\end{array}$ & $\begin{array}{c}\Phi \text { Cancelation } \\
\text { Rates } 10^{18} \\
\mathrm{Mx} \mathrm{hr}\end{array}$ \\
\hline$\overline{\mathrm{A}}$ & $1(\mathrm{~A} 1)$ & $22: 07$ & No & $\ldots$ & $11,000 \pm 500$ & $\ldots$ & $2300 \pm 500$ & $23 \pm 1$ & $4 \pm 12 \mathrm{~s}$ & $3200 \pm 500$ & $\ldots$ & 1.0 \\
\hline \multirow[t]{2}{*}{ B } & 2 (B1) & 13:57, & No & $\ldots$ & $\ldots \mathrm{j}$ & $\ldots$ & $\ldots$ & $\ldots$ & $2 \pm 24 \mathrm{~s}$ & $2200 \pm 500$ & $\ldots$ & $\ldots{ }^{\mathrm{j}}$ \\
\hline & (B2) & $19: 04^{\mathrm{k}}$ & Yes & $6900 \pm 800$ & $\ldots$ & $800 \pm 70$ & $\ldots$ & $\ldots$ & $2 \pm 12 \mathrm{~s}$ & $2100 \pm 200$ & $1000 \pm 80$ & $\ldots$ \\
\hline \multirow[t]{3}{*}{$\mathrm{C}$} & $3(\mathrm{C} 1)$ & 16:40, & No & $\cdots$ & $25,000 \pm 2500$ & $\ldots$ & $5000 \pm 25$ & $110 \pm 25$ & $2 \pm 12 \mathrm{~s}$ & $2500 \pm 400$ & $\ldots$ & 1.7 \\
\hline & (C2) & 18:33, & Yes & $19,000 \pm 1000$ & $\ldots^{1}$ & $1300 \pm 300$ & & $\ldots^{1}$ & $3 \pm 24 s$ & $3000 \pm 300$ & $4200 \pm 300$ & $" 1$ \\
\hline & (C3) & $19: 15$ & Yes & $23,000 \pm 1000$ & $33,000 \pm 1800$ & $2100 \pm 200$ & $2500 \pm 600$ & $120 \pm 50$ & $4 \pm 24 \mathrm{~s}$ & $5000 \pm 800$ & $3000 \pm 200$ & " \\
\hline $\mathrm{D}$ & 1 (D1) & $19: 15$ & Yes & $11,000 \pm 800$ & $18000 \pm 1500$ & $4000 \pm 300$ & $1500 \pm 400$ & $50 \pm 20$ & $3 \pm 12 \mathrm{~s}$ & $4300 \pm 200$ & $6000 \pm 250$ & 2.6 \\
\hline \multirow[t]{3}{*}{$\mathrm{E}$} & 3 (E1) & $21: 16$ & Yes & $16500 \pm 300$ & $33,000 \pm 3500$ & $7000 \pm 100$ & $2200 \pm 150$ & $70 \pm 30$ & $3 \pm 12 \mathrm{~s}$ & $10,000 \pm 1000$ & $8000 \pm 100$ & 0.6 \\
\hline & (E2) & $22: 37$ & No & $\ldots$ & $32000 \pm 2000$ & $\ldots$ & $2000 \pm 150$ & $60 \pm 10$ & $4 \pm 24 \mathrm{~s}$ & $5000 \pm 300$ & $\ldots$ & " \\
\hline & (E3) & $23: 14$ & No & $\ldots$ & $33600 \pm 1200$ & $\cdots$ & $7000 \pm 150$ & $50 \pm 10$ & $5 \pm 12 \mathrm{~s}$ & $6500 \pm 200$ & $\ldots$ & $"$ \\
\hline Average $\pm 1 \sigma_{\text {ave }}$ & & & & $16,000 \pm 6000$ & $27,000 \pm 8000$ & $3000 \pm 2500$ & $3200 \pm 2000$ & $70 \pm 30$ & $3 \pm 1$ & $4200 \pm 2200$ & $4400 \pm 2500$ & 1.5 \\
\hline
\end{tabular}

Notes.

a Jetlet locations in Figure 1(c).

${ }^{\mathrm{b}}$ Total number of jetlets from the same neutral line.

${ }^{c}$ Approximate time of brightening at the base of jetlets in AIA $171 \AA$ images.

${ }^{d}$ Maximum length of the spire from base to the visible tip of the spire.

e Width measured in the middle of the spire using AIA $171 \AA$ A and IRIS Si IV SJI.

${ }^{f}$ Plane-of-sky speed along the jetlet spire. Speeds and uncertainties are measured from AIA $171 \AA$ time-distance maps.

g Duration of jetlet spire in AIA $171 \AA$ A images.

${ }^{\mathrm{h}}$ Cross-sectional width of the jetlet base during the jetlet onset in AIA $171 \AA$ A and IRIS Si IV SJI.

${ }^{1}$ Average flux cancelation rates from $1-2 \mathrm{hr}$ before the jetlet to $0-1 \mathrm{hr}$ after the jetlet.

${ }^{\mathrm{j}}$ Jetlet is barely visible in AIA images. HMI magnetograms do not show minority-polarity flux at the base of the jetlet.

${ }^{\mathrm{k}}$ There are small-scale jetlets around that time in IRIS images.

${ }^{1}$ Spire is too faint in AIA images to estimate its length, width, and speed. 

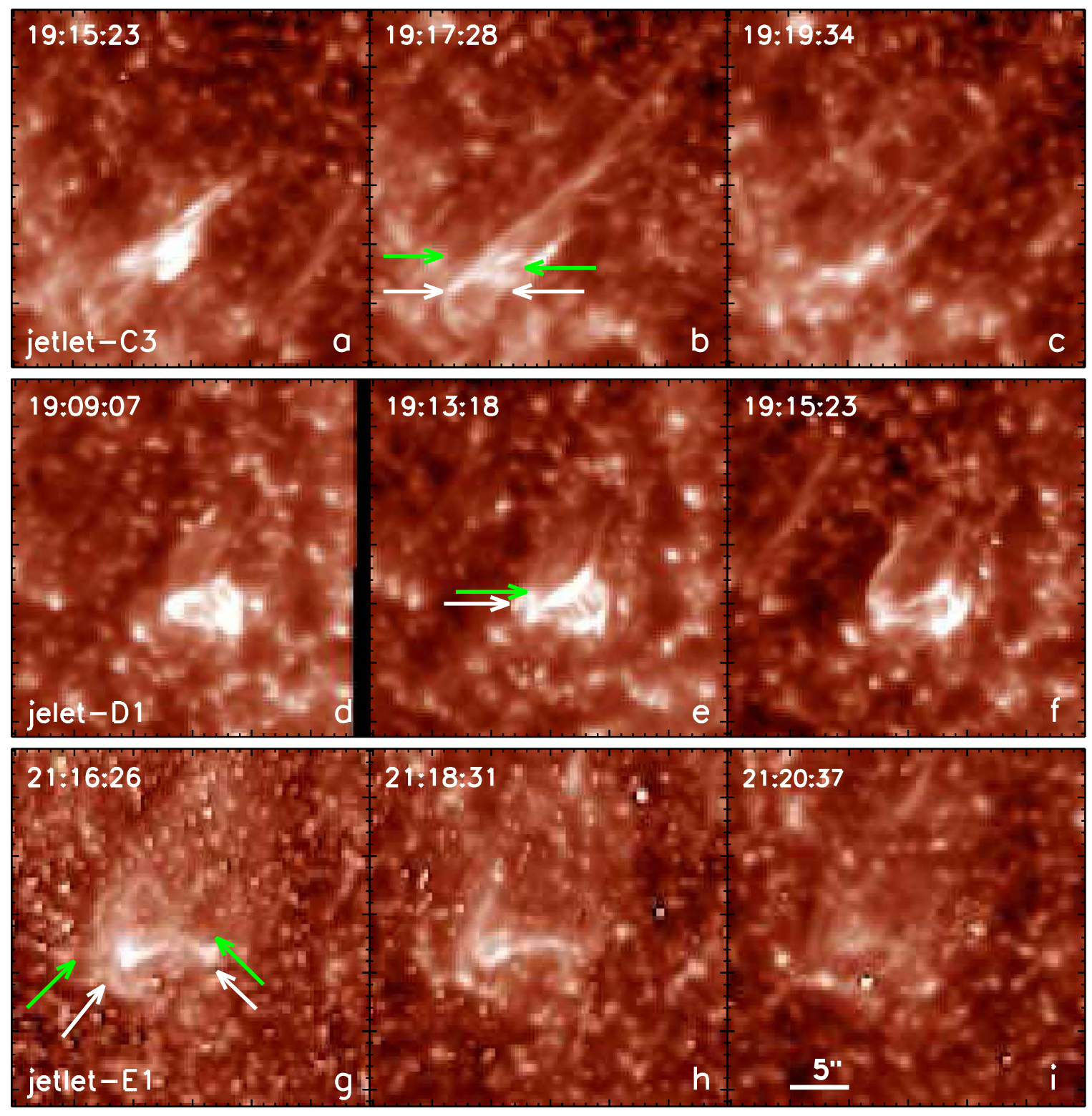

Figure 2. IRIS Si IV SJIs of the evolution of three jetlets. Panels (a)-(c) show a jetlet (C3) from location C of Figure 1. Panels (d)-(e) show a jetlet (D1) from location D of Figure 1. Panels (g)-(i) show a jetlet (E1) from location E of Figure 1. The white arrows point to the Si IV brightenings at the base of $171 \AA$ Alare loops. The green arrows point to the locations of the feet of the bright loops in $171 \AA$ images. In the animation, the temporal cadence of each frame is $\sim 2$ minutes.

(An animation of this figure is available.)

measured the minority-polarity flux patch (negative) because it is well isolated within the box (Figure 4(a)). The negative flux decreases, which is a clear indication of flux cancelation at the base of the jetlet. We surmise that the continuous flux cancelation over $\sim 6 \mathrm{hr}$ prepares and eventually triggers each of these three sequential eruptions. After the first jetlet, a significant amount of flux still remains, and that flux continues to cancel before the second homologous eruption and further cancelation apparently prepares and triggers a third homologous eruption (the negative-flux bump at 19:17 UT is from coalescence of weak flux grains unrelated to the jetlet). We estimate the average rate of flux decrease using the best-fit line in Figure 4(c) and find it to be $\sim 1.7 \times 10^{18} \mathrm{Mx} \mathrm{hr}^{-1}$. Miniflaments in sequential CJs have also been observed to erupt and reform/reappear at the same PIL due to flux cancelation (Panesar et al. 2017).

\subsection{The Jetlet from Region $D$}

Figures 2(d)-(f) and 3(d)-(f) show the single jetlet in region D. The photospheric magnetic field in region $\mathrm{D}$ is displayed in Figures 4(d) and (e). Base brightening starts at 19:09 UT (Figures 2(d) and 3(d)). At 19:12 UT the spire extends outward with an average speed of $50 \pm 20 \mathrm{~km} \mathrm{~s}^{-1}$, and the total duration of the spire is 3 minutes. The green arrow in Figure 3(e) points to the AIA $171 \AA$ brightening that appeared at the PIL during the onset. In the Si IV SJIs, the base brightenings appear lower down (Figure 2(e)) in comparison to the base brightenings in the AIA $171 \AA$ images (this is the case 

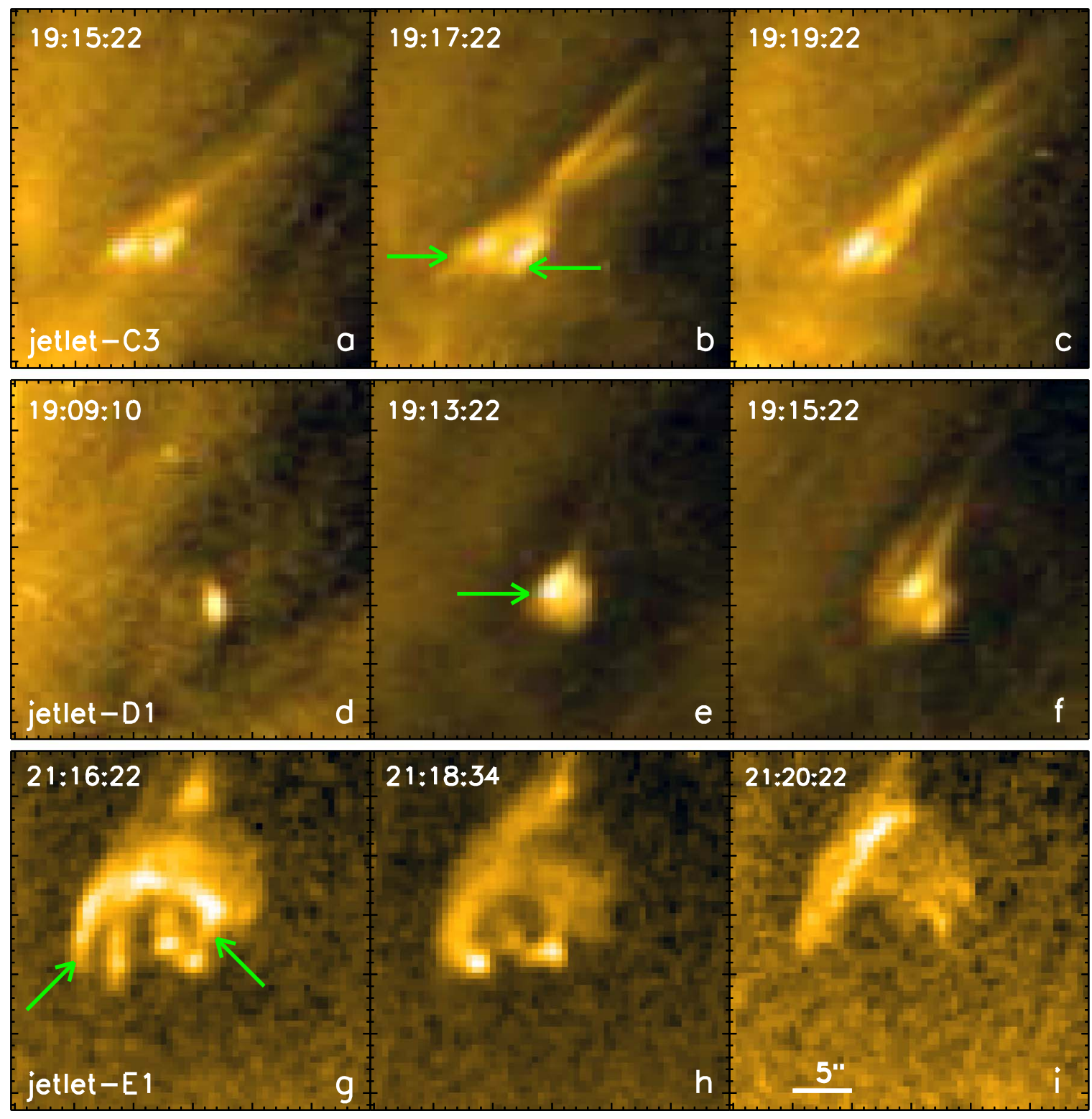

Figure 3. AIA $171 \AA$ images of the evolution of the three jetlets of Figure 2. The format is similar to that of Figure 2. The green arrows point to the feet of bright loops. In the animation, the temporal cadence of each frame is $\sim 1$ minutes.

(An animation of this figure is available.)

in all 10 jetlets). The Si IV images show transition-regiontemperature plasma whereas the $171 \AA$ images show relatively hotter coronal plasma.

There is an emergence of a bipole, at $~ 18: 52 \mathrm{UT}$, next to the majority-polarity (positive) network flux lane (MOVIE3b). One foot (negative polarity) of the newly emerged bipole starts merging and canceling with the neighboring majority-polarity network flux lane (Figures 4(d) and (e)). The flux cancelation between the foot of the newly emerged bipole and pre-existing flux results in the jetlet at 19:09 UT. This is analogous to CJs, where a pre-jet minifilament forms and erupts due to flux cancelation between one foot of a newly emerged bipole and a pre-existing majority-polarity flux patch (e.g., Panesar et al. 2017).

Figure 4(f) shows a minority-polarity flux-versus-time plot for region D. First, there is an increase in the negative flux, due to the flux emergence ( $\sim 20$ minutes) before the jetlet onset. Later, at 19:00 UT, negative flux starts to decrease; the flux cancelation triggers the jetlet eruption. The flux cancels with an average rate of $\sim 2.6 \times 10^{18} \mathrm{Mx} \mathrm{hr}^{-1}$.

\subsection{Jetlets from Region $E$}

We show our third example jetlet, E1, in Figures 2(g)-(i) and Figures 3(g)-(i). We observed three (homologous) jetlets within a period of two hours from the same PIL. During the onset of jetlet E1, we observe brightenings of base loops (Figures 2 and 3), which link opposite-polarity flux patches (Figure 4).

Figures $4(\mathrm{~g})$, (h) show the magnetic field before and after the jetlet onset. There is a decrease in negative flux in the jetletbase region (Figure 4(i)), which indicates that flux cancelation is the trigger of sequential/homologous jetlets. The HMI animation (MOVIE3c) shows that opposite-polarity flux patches approach toward the PIL, and apparently flux cancelation between them triggers the first jetlet (E1) at 

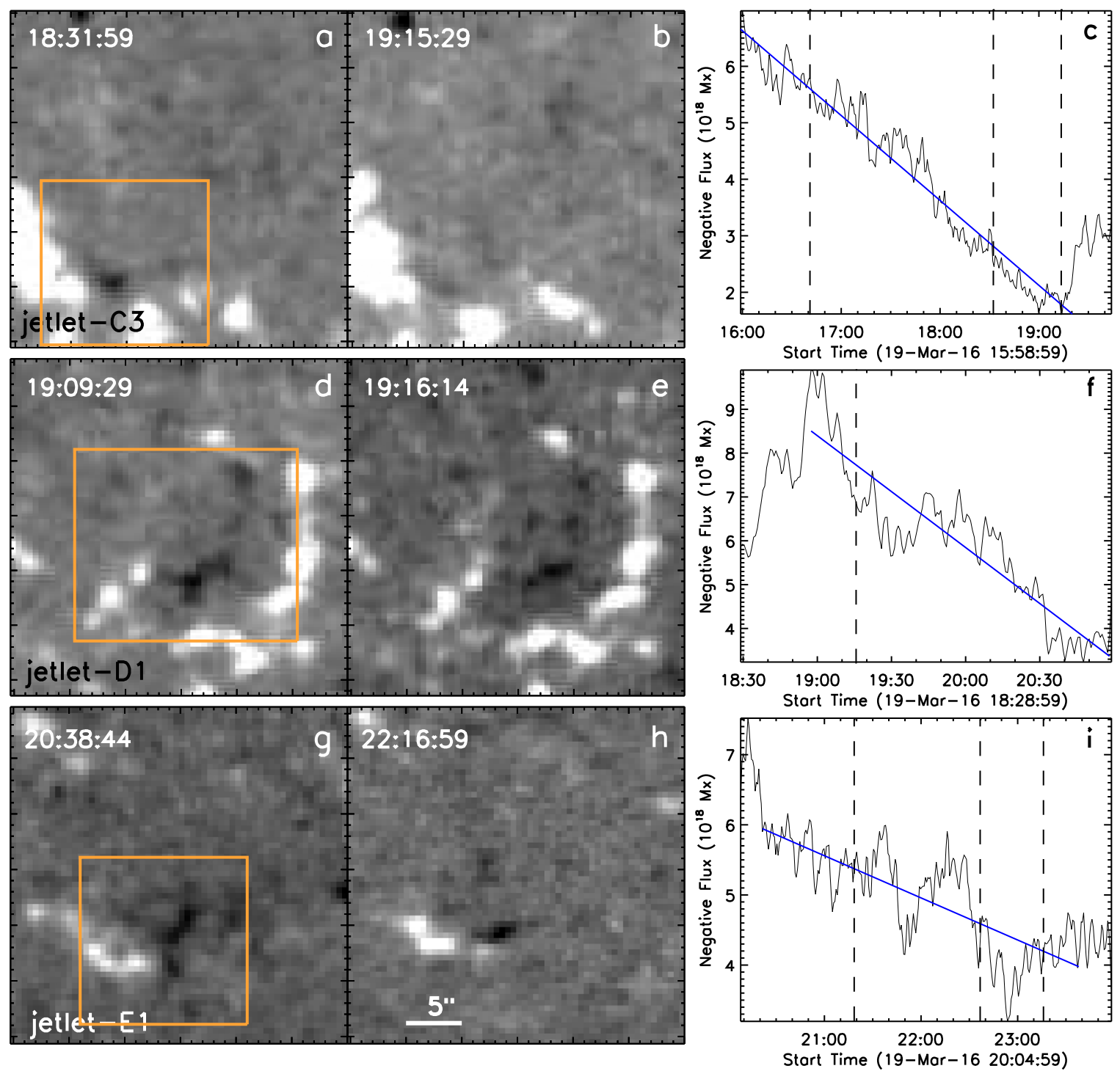

Figure 4. HMI magnetograms of the three jetlet locations. Panels (a)-(b), (d)-(e), and (g)-(i) show the magnetic field near the base of jetlets C3, D1, and E1, respectively. The orange boxes in Figures 4(a), (d), and (g) show the regions measured for the magnetic flux time plots in Figures 4(c), (f), and (i), respectively (negative flux in each case). The blue line in Figures 4(c), (f), and (i) is a least-square fit from before to after the jetlet. The dashed lines show jetlet onset times. In the animation of the a-b, d-e, and g-h panels, the temporal cadence of each frame is $\sim 45 \mathrm{~s}$.

(An animation of this figure is available.)

21:16 UT (Figure 4(i)). The flux continues to cancel after the first jetlet and continues until the minority-polarity flux patch is almost all gone (MOVIE3c). The continuous flux cancelation leads to the second (E2) and third (E3) homologous jetlets at 22:37 UT and 23:14 UT, respectively. Eventually, the minority-polarity patch is nearly gone and jetting stops. The average flux cancelation rate is $\sim 0.6 \times 10^{18} \mathrm{Mx} \mathrm{hr}^{-1}$.

\section{Discussion}

We have examined the magnetic environment of 10 on-disk jetlets in UV/EUV in a coronal-hole network region using IRIS and $S D O$ data, and find that jetlets have many similarities with typical CJs. Recent observations of CJs (e.g., Huang et al. 2012; Shen et al. 2012; Panesar et al. 2016b, 2018; Sterling et al. 2017) show that flux cancelation is usually the trigger of CJs. Often continuous flux cancelation leads to recurrent/ homologous jets (Chandra et al. 2015; Sterling et al. 2016;
Panesar et al. 2017). CJs show base brightenings during the onset and the spire is frequently seen to extend along a twisting magnetic field (e.g., Schmieder et al. 2013; Moore et al. 2015; Panesar et al. 2016a). Our observations show that jetlets share these properties of CJs, and hence are plausibly scaled-down versions of CJs. Here we summarize our findings:

Flux cancelation: We find that all 10 of our jetlets occur at the edges of magnetic network flux lanes, all but 1 of them at a site of apparent magnetic flux cancelation. The continuous flux cancelation builds and triggers the homologous jetlet eruptions from regions $\mathrm{B}, \mathrm{C}$, and $\mathrm{E}$. The 1 exception (out of 10 jetlets) is jetlet $\mathrm{B} 1$, where HMI magnetograms do not show a minoritypolarity flux patch at the jetlet base in region $\mathrm{B}$. There are two possibilities in this case: either there is no minority-polarity flux present in this region, or minority-polarity flux is there but too weak to be detected by HMI. We tried increasing the HMI magnetogram sensitivity using a post-launch-improved estimate for the instrumental point-spread function, as described in 
Couvidat et al. (2016), but we were still unable to detect minority polarity at the jetlet base in region $\mathrm{B}$.

The average flux cancelation rate for our nine jetlets having obvious cancelation is $\sim 1.5 \times 10^{18} \mathrm{Mx} \mathrm{hr}^{-1}$, which is similar to that for CJs in quiet regions $\left(\sim 1.5 \times 10^{18} \mathrm{Mx} \mathrm{hr}^{-1}\right.$; Panesar et al. 2016b) and coronal holes $\left(\sim 0.6 \times 10^{18} \mathrm{Mx} \mathrm{hr}^{-1}\right.$; Panesar et al. 2018), whereas active regions CJs have higher flux cancelation rates $\sim 1.5 \times 10^{19} \mathrm{Mx} \mathrm{hr}^{-1}$ (Sterling et al. 2017).

Base brightenings: During the eruption onset, we observe brightenings at the base of each of the jetlets. These brightenings occur at the PIL where opposite-polarity flux patches cancel. Jetlet-base brightenings include brightenings that might be miniatures of the JBP that is seen to occur at the flux cancelation PIL in CJs during minifilament-eruption onset.

Twist in a jetlet spire: In one jetlet (C3), we observe possible twisting motion in the jetlet spire, which could be the result of a miniature erupting flux rope having external reconnection with the ambient open field. We do not see a "microfilament" of the size of the jetlet base (Sterling \& Moore 2016) in the observations. If jetlets do have "microfilament" flux ropes at their PILs before the eruption, then they plausibly work in the same way as CJs and CMEs. They may be visible in higherspatial-resolution data from current/future instruments, e.g., IRIS spectroheliograms, SST, GST, Gregor, DKIST, and Solar-C.

Comparison between the properties of CJs and UV/EUV jetlets: Jetlets shoot out with an average speed of $70 \pm 30 \mathrm{~km} \mathrm{~s}^{-1}$, which is similar to the average speeds of quiet-region $\left(100 \pm 20 \mathrm{~km} \mathrm{~s}^{-1}\right)$ and coronal-hole $\left(70 \pm 30 \mathrm{~km} \mathrm{~s}^{-1}\right)$ jets estimated by Panesar et al. (2016b, 2018). The average duration of jetlets is four times shorter $(3 \pm 1 \mathrm{~m})$ than the typical duration of CJs ( 12 minutes Shimojo et al. 1996; Savcheva et al. 2007; Panesar et al. 2016b, 2018). Using AIA $171 \AA$ images (IRIS Si IV SJI), we estimated that jetlet-base width, spire length, and spire width have mean values of about $4200 \pm 2500 \mathrm{~km} \mathrm{(4400 \pm 2500}$ $\mathrm{km}), 27,000 \pm 8000 \mathrm{~km}(16,000 \pm 6000 \mathrm{~km})$, and $3200 \pm 2000$ $\mathrm{km}(3000 \pm 2500 \mathrm{~km})$, respectively. The studied jetlets, on average, are at least three times smaller in base width $(<5000 \mathrm{~km})$ than typical CJs $(\sim 18,000 \mathrm{~km})$.

Comparison with spicules: The observed jetlet speeds and durations are similar to Type-II spicule speeds $\left(30-110 \mathrm{~km} \mathrm{~s}^{-1}\right)$ and durations $(0.83-2.5 \mathrm{~m})$, but their spire widths are six times larger than spicule widths $(500 \mathrm{~km}$; Sterling 2000; De Pontieu et al. 2007; Pereira et al. 2012). Also, the occurrence rate of jetlets (some tens in $24 \mathrm{hr}$ in the Figure 1(b) FOV), is much less than that of spicules (e.g., Beckers 1972).

Jetlets show some similarities with spicules: Both features occur at the edges of network lanes, have similar velocities and durations, and can show twisting motions. However, the coronal response of jetlets is different from that of spicules. So it could be that jetlets are on the smaller-size-scale end of features driven like CJs (see Sterling \& Moore 2016), while spicule might have a different driving mechanism with natural explanations for their velocities, durations, and twisting motions (e.g., Iijima \& Yokoyama 2017; Martínez-Sykora et al. 2017).

Our recent CJ observations (Sterling et al. 2015; Panesar et al. 2016b) apparently show (a) a pre-eruption minifilament sits at a PIL of a sheared bipole between a minority-polarity flux patch and majority-polarity flux patch; (b) due to continuous flux cancelation at the PIL, the minifilament field eventually becomes unstable and erupts outward and a JBP appears at the PIL via internal reconnection; (c) the outer envelope of the erupting minifilament field reconnects with the neighboring open field, which results in the formation of new open field lines and CJ material flowing out along these newly opened lines.

Our jetlet observations appear to be consistent with the CJ picture in some ways: Jetlets occur at PILs between merging minority and majority flux patches, the minority-polarity flux patch approaches (and cancels with) the majority-polarity network flux patch, and the flux cancelation plausibly prepares and triggers one or more small-scale flux rope eruptions that drive one or more jetlets. One of our jetlet spires (C3) seems to show twisting motion, which suggests that a highly twisted flux rope erupted from the jetlet base. The erupting small-scale flux rope would result in internal reconnection in the erupting field, and brightenings/JBP (Figures 2 and 3) appear at the eruption site. The spire would start to form when the erupting flux rope reconnects (external reconnection) with encountered open field. The jetlet material would then escape along the newly opened field lines. We do not, however, see any clear signatures of brightenings of closed loops made by external reconnection of the erupting field with the encountered open field as in the Sterling et al. (2015) CJ picture; we need further observations to determine whether this is due to the brightenings being too faint to observe with IRIS and AIA, or because there is an inconsistency with the CJ picture.

\section{Conclusion}

Our observations of 10 jetlets suggest that flux cancelation is a necessary condition for the buildup and triggering of UV/EUV network jetlets and they usually stem from the edges of magnetic network flux lanes. Jetlets are therefore plausibly small-scale versions of the larger CJs, and of still-larger CME events.

N.K.P.'s research was supported by an appointment to NPP at the NASA/MSFC, administered by USRA under contract with NASA. A.C.S. and R.L.M. acknowledge support from the NASA HGI program, and by the Hinode Project. S.K.T. acknowledges support by NASA contract NNG09FA40C (IRIS). We acknowledge the use of IRIS and SDO data. B.D.P. acknowledges support from NASA grants NNX16AG90G and NNG09FA40C (IRIS). IRIS is a NASA small explorer mission developed and operated by LMSAL with mission operations executed at NASA Ames Research center and major contributions to downlink communications funded by ESA and the Norwegian Space Centre.

\section{ORCID iDs}

Navdeep K. Panesar (1) https://orcid.org/0000-00017620-362X

Alphonse C. Sterling $\odot$ https://orcid.org/0000-00031281-897X

Ronald L. Moore (i) https://orcid.org/0000-0002-5691-6152 Sanjiv K. Tiwari i https://orcid.org/0000-0001-7817-2978 Bart De Pontieu (ํ) https://orcid.org/0000-0002-8370-952X Aimee A. Norton $\odot$ https://orcid.org/0000-0003-2622-7310

\section{References}

Adams, M., Sterling, A. C., Moore, R. L., \& Gary, G. A. 2014, ApJ, 783, 11 Avallone, E. A., Tiwari, S. K., Panesar, N. K., Moore, R. L., \& Winebarger, A. 2018, ApJ, 861, 111 
Beckers, J. M. 1972, ARA\&A, 10, 73

Chandra, R., Gupta, G. R., Mulay, S., \& Tripathi, D. 2015, MNRAS, 446, 3741

Couvidat, S., Schou, J., Hoeksema, J. T., et al. 2016, SoPh, 291, 1887

De Pontieu, B., Erdélyi, R., \& James, S. P. 2004, Natur, 430, 536

De Pontieu, B., McIntosh, S., Hansteen, V. H., et al. 2007, PASJ, 59, 655

De Pontieu, B., McIntosh, S. W., Carlsson, M., et al. 2011, Sci, 331, 55

De Pontieu, B., Title, A. M., Lemen, J. R., et al. 2014, SoPh, 289, 2733

Dodson-Prince, H. W., \& Bruzek, A. 1977, in Illustrated Glossary for Solar and

Solar-Terrestrial Physics, ed. A. Bruzek \& C. J. Durrant (Dordrecht: Springer Netherlands), 81

Hong, J., Jiang, Y., Zheng, R., et al. 2011, ApJL, 738, L20

Huang, Z., Madjarska, M. S., Doyle, J. G., \& Lamb, D. A. 2012, A\&A, 548, A62

Iijima, H., \& Yokoyama, T. 2017, ApJ, 848, 38

Innes, D. E., Cameron, R. H., \& Solanki, S. K. 2011, A\&A, 531, L13

Lemen, J. R., Title, A. M., Akin, D. J., et al. 2012, SoPh, 275, 17

Martin, S. F. 1973, SoPh, 31, 3

Martínez-Sykora, J., De Pontieu, B., Hansteen, V. H., et al. 2017, Sci, 356, 1269

Moore, R. L., Falconer, D. A., Porter, J. G., \& Suess, S. T. 1999, SSRv, 87,283

Moore, R. L., Sterling, A. C., \& Falconer, D. A. 2015, ApJ, 806, 11

Moore, R. L., Sterling, A. C., Panesar, N. K., et al. 2018, ApJ, 859, 3

Panesar, N. K., Sterling, A. C., \& Moore, R. L. 2016a, ApJL, 822, L23
Panesar, N. K., Sterling, A. C., \& Moore, R. L. 2017, ApJ, 844, 131

Panesar, N. K., Sterling, A. C., \& Moore, R. L. 2018, ApJ, 853, 189

Panesar, N. K., Sterling, A. C., Moore, R. L., \& Chakrapani, P. 2016b, ApJL, 832, L7

Pereira, T. M. D., De Pontieu, B., \& Carlsson, M. 2012, ApJ, 759, 18

Raouafi, N. E., Patsourakos, S., Pariat, E., et al. 2016, SSRv, 201, 1

Raouafi, N.-E., \& Stenborg, G. 2014, ApJ, 787, 118

Savcheva, A., Cirtain, J., Deluca, E. E., et al. 2007, PASJ, 59, 771

Schmieder, B., Guo, Y., Moreno-Insertis, F., et al. 2013, A\&A, 559, A1

Schou, J., Scherrer, P. H., Bush, R. I., et al. 2012, SoPh, 275, 229

Shen, Y., Liu, Y., Su, J., \& Deng, Y. 2012, ApJ, 745, 164

Shibata, K., Ishido, Y., Acton, L. W., et al. 1992, PASJ, 44, L173

Shimojo, M., Hashimoto, S., Shibata, K., et al. 1996, PASJ, 48, 123

Sterling, A. C. 2000, SoPh, 196, 79

Sterling, A. C., \& Moore, R. L. 2016, ApJL, 828, L9

Sterling, A. C., Moore, R. L., Falconer, D. A., et al. 2016, ApJ, 821, 100

Sterling, A. C., Moore, R. L., Falconer, D. A., \& Adams, M. 2015, Natur, 523,437

Sterling, A. C., Moore, R. L., Falconer, D. A., Panesar, N. K., \& Martinez, F. 2017, ApJ, 844, 28

Wang, Y.-M., Sheeley, N. R., Jr., Socker, D. G., et al. 1998, ApJ, 508, 899

Zirker, J. B. 1989, SoPh, 119, 341 